\author{
MITSUBISHI ELECTRIC RESEARCH LABORATORIES \\ http://www.merl.com
}

\title{
Observer Forms for Perspective Systems
}

\author{
Ola Dahl, Yebin Wang, Alan F. Lynch, Anders Heyden
}

TR2010-117 December 2010

\begin{abstract}
Estimation of 3D position information from 2D images in computer vision systems can be formulated as a state estimation problem for a nonlinear perspective dynamic system. The multioutput state estimation problem has been treated by several authors using methods for nonlinear observer design. This paper shows that a perspective system can be transformed to two observer forms, and provides constructive methods for arriving at the transformations. These observer forms lead to straightforward observer designs. First, it is shown that using an output transformation, the system admits an observer form which leads to an observer with linear error dynamics. A second observer design is based on a time scaled block triangular form. Both designs assume a commonly used observability condition. The designs are demonstrated in simulation.
\end{abstract}

Automatica

This work may not be copied or reproduced in whole or in part for any commercial purpose. Permission to copy in whole or in part without payment of fee is granted for nonprofit educational and research purposes provided that all such whole or partial copies include the following: a notice that such copying is by permission of Mitsubishi Electric Research Laboratories, Inc.; an acknowledgment of the authors and individual contributions to the work; and all applicable portions of the copyright notice. Copying, reproduction, or republishing for any other purpose shall require a license with payment of fee to Mitsubishi Electric Research Laboratories, Inc. All rights reserved.

Copyright (C) Mitsubishi Electric Research Laboratories, Inc., 2010

201 Broadway, Cambridge, Massachusetts 02139 



\title{
Observer Forms for Perspective Systems *
}

\author{
Ola Dahl ${ }^{\text {a }}$ Yebin Wang ${ }^{\mathrm{b}}$ Alan F. Lynch ${ }^{\mathrm{c}}$ Anders Heyden ${ }^{\mathrm{d}}$ \\ ${ }^{a} D D A$ Consulting AB, Malmö, Sweden,e-mail: ola.dahl@ddacon.se \\ ${ }^{\mathrm{b}}$ Mitsubishi Electric Research Laboratories, Cambridge, MA, e-mail: yebinwang@ieee.org \\ ${ }^{\mathrm{c}}$ Dept. of Electrical 85 Computer Engineering, University of Alberta, Edmonton, AB, Canada, e-mail: alanl@ieee.org \\ ${ }^{\mathrm{d}}$ Dept. Applied Mathematics, Lund University, Lund, Sweden, e-mail: heyden@maths.lth.se
}

\begin{abstract}
Estimation of $3 \mathrm{D}$ position information from 2D images in computer vision systems can be formulated as a state estimation problem for a nonlinear perspective dynamic system. The multi-output state estimation problem has been treated by several authors using methods for nonlinear observer design. This paper shows that a perspective system can be transformed to two observer forms, and provides constructive methods for arriving at the transformations. These observer forms lead to straightforward observer designs. First, it is shown that using an output transformation, the system admits an observer form which leads to an observer with linear error dynamics. A second observer design is based on a time scaled block triangular form. Both designs assume a commonly used observability condition. The designs are demonstrated in simulation.
\end{abstract}

\section{Introduction}

The problem of estimating $3 \mathrm{D}$ structure and motion from 2D perspective observations can be solved with a nonlinear observer design. The perspective system dynamics is obtained by considering the relative motion between a perspective camera and an observed object. The estimation of both structure and motion can be achieved by an observer for states and parameters. Existing approaches have used the extended Kalman filter [17] or adaptive observers $[3,6]$. The problem of estimating structure when the motion parameters are measured or otherwise assumed available, has been considered using observer-based approaches in $[15,10,16,2,9,7,1,11]$.

Based on preliminary results in [8], this paper presents structure estimation results by showing how a perspective system can be transformed into two observer forms. These forms naturally lead to observers with simple error dynamics systems. The simplicity of the error dynamics leads to a straightforward stability analysis. Relative to existing related work, the results here show that it is possible to achieve linear time-invariant error dynamics without any constraints on the type of motion when an Observer Form (OF) with output transformation is considered [13]. Previous work in [5] considered

\footnotetext{
^ This work was supported in part by the Natural Sciences and Engineering Research Council of Canada (NSERC) under grant number \#249681.
}

the $\mathrm{OF}$ without output transformation, and required a constraint on the type of motion which potentially limited the application of the approach. A second contribution of the paper is to demonstrate the application of a Time-Scaled Block Triangular Observer Form (TBTOF) which was first introduced in [21]. The TBTOF is a generalization of $\mathrm{OF}$ and can therefore be applied to a wider class of systems. The advantage of the TBTOF is a simpler observer structure since it leaves part of the dynamics untransformed.

\section{Background}

\subsection{Perspective dynamic systems}

A perspective dynamic system with three states and two outputs, derived assuming a calibrated pinhole camera and observations of feature points on a rigid object, can be written as

$$
\dot{\zeta}=\left[\begin{array}{lll}
a_{11} & a_{12} & a_{13} \\
a_{21} & a_{22} & a_{23} \\
a_{31} & a_{32} & a_{33}
\end{array}\right] \zeta+\left[\begin{array}{l}
b_{1} \\
b_{2} \\
b_{3}
\end{array}\right], y=\left[\begin{array}{lll}
\zeta_{1} / \zeta_{3} & \zeta_{2} / \zeta_{3}
\end{array}\right]^{T},
$$

where we assume $a_{i j}, b_{i}, 1 \leq i, j \leq 3$ are constant and $\zeta_{3}>0[14]$. 
As in e.g. [2], it is convenient to work in state coordinates defined by $\xi=\left[\xi_{1}, \xi_{2}, \xi_{3}\right]^{T}=\left[\zeta_{1} / \zeta_{3}, \zeta_{2} / \zeta_{3}, 1 / \zeta_{3}\right]^{T}$ which transforms (1) into

$$
\begin{aligned}
\dot{\xi}_{1}= & \left(a_{11}-a_{33}\right) \xi_{1}+a_{12} \xi_{2}+a_{13}+\left(b_{1}-b_{3} \xi_{1}\right) \xi_{3} \\
& -a_{31} \xi_{1}^{2}-a_{32} \xi_{1} \xi_{2} \\
\dot{\xi}_{2}= & a_{21} \xi_{1}+\left(a_{22}-a_{33}\right) \xi_{2}+a_{23}+\left(b_{2}-b_{3} \xi_{2}\right) \xi_{3} \\
& -a_{31} \xi_{2} \xi_{1}-a_{32} \xi_{2}{ }^{2} \\
\dot{\xi}_{3}= & -\xi_{3}\left(a_{31} \xi_{1}+a_{32} \xi_{2}+a_{33}+b_{3} \xi_{3}\right) \\
y= & {\left[\begin{array}{ll}
\xi_{1} & \xi_{2}
\end{array}\right]^{T} . }
\end{aligned}
$$

In the $\xi$ coordinates the dynamics are nonlinear and the output function is linear.

In the following we use the notation $L_{f} h$ for the Lie derivative of a function $h: \mathbb{R}^{n} \rightarrow \mathbb{R}$ along a vector field $f: \mathbb{R}^{n} \rightarrow \mathbb{R}^{n}$ and $L_{f}^{k} h(x)$ for the $k$ times repeated Lie derivative. The notation $d h$ is used for the gradient of a function $h: \mathbb{R}^{n} \rightarrow \mathbb{R}$. Given vector fields $f, g: \mathbb{R}^{n} \rightarrow$ $\mathbb{R}^{n}, a d_{f} g$ denotes the Lie bracket $[f, g]$ and $a d_{f}^{i} g$ is the repeated Lie bracket $a d_{f}^{i} g=\left[f, a d_{f}^{i-1} g\right]$ and $a d_{f}^{0} g=g$.

\subsection{Observability}

In order to apply the definition of observability in [13] we compute

$$
\Omega=\left[\begin{array}{c}
d h_{1} \\
d h_{2} \\
d L_{f} h_{1} \\
d L_{f} h_{2}
\end{array}\right]=\left[\begin{array}{ccc}
1 & 0 & 0 \\
0 & 1 & 0 \\
* * & b_{1}-b_{3} \xi_{1} \\
* & * & b_{2}-b_{3} \xi_{2}
\end{array}\right] .
$$

We note the codistribution $\Omega$ has dimension 3 and system $(2)$ is observable with observability indices $k_{1}=$ $2, k_{2}=1$ provided $\left(b_{1}-b_{3} \xi_{1}\right)^{2}+\left(b_{2}-b_{3} \xi_{2}\right)^{2} \neq 0$. The value of the output $y=\left[b_{1} / b_{3}, b_{2} / b_{3}\right]^{T}$ where the system loses observability is called the focus of expansion [10]. In this paper, we assume $b_{1}-b_{3} \xi_{1} \neq 0$ so that $h_{1}, h_{2}, L_{f} h_{1}$ are coordinates. The constraint $b_{1}-b_{3} \xi_{1} \neq$ 0 in the approach proposed here can be generalized to $c_{1}\left(b_{1}-b_{3} \xi_{1}\right)+c_{2}\left(b_{2}-b_{3} \xi_{2}\right) \neq 0$ for some $c_{1}, c_{2} \in \mathbb{R}$.

\section{Observer forms for perspective systems}

Without output transformation it was shown in [5] that (1) admits an OF under the constraint $b_{2}=b_{3}=0$ given the observability assumption $b_{1}-b_{3} \xi_{1} \neq 0$. In this paper we provide two results which extend [5]. The first result shows the existence of an output transformation $\bar{y}=\Psi(y)$ and state transformation $z=\Phi(\xi)$ such that (1) is transformable to OF with weaker motion constraints relative to [5]. The second result demonstrates the existence of a TBTOF which provides coordinates allowing for a straightforward observer design, albeit with the same constraint on the motion which appeared in [5] for dynamic error linearization. The results were derived with the help of a Maple library for observer error linearization [4].

\subsection{Observer Form with output transformation}

We have the following theorem which provides the necessary and sufficient conditions for transformation to $\mathrm{OF}$ without output transformation.

Theorem 1 ([22]) The dynamic system

$$
\dot{\zeta}=f(\zeta), \quad y=h(\zeta)
$$

where $\zeta \in \mathbb{R}^{n}, y \in \mathbb{R}^{2}$, is locally transformable to $O F$ with the change of state coordinates $z=\Phi(\zeta)$ if and only if the following three conditions are locally satisfied:

(i) The codistributions

$$
\begin{gathered}
R_{j}^{l}=\operatorname{span}\left\{d L_{f}^{k} h_{i}: 0 \leq k \leq k_{j}-1, i \neq j\right. \\
\left.\left.1 \leq i \leq 2, d L_{f}^{k} h_{j}: 0 \leq k \leq k_{j}-2\right)\right\} \\
R_{j}^{r}=\operatorname{span}\left\{d L_{f}^{k} h_{i}: 0 \leq k \leq \min \left(k_{i}, k_{j}\right)-1, i \neq j\right. \\
\left.1 \leq i \leq 2, d L_{f}^{k} h_{j}: 0 \leq k \leq k_{j}-2\right\}
\end{gathered}
$$

satisfy $\operatorname{dim} R_{j}^{l}=\operatorname{dim} R_{j}^{r}, 1 \leq j \leq 2$.

(ii) There exist vector fields $r_{i}, 1 \leq i \leq 2$, such that

$$
\begin{aligned}
& L_{r_{i}} L_{f}^{k-1} h_{j}=\delta_{i, j} \cdot \delta_{k, k_{j}} \\
& 1 \leq i \leq 2, \quad 1 \leq k \leq k_{i}, \quad 1 \leq j \leq 2
\end{aligned}
$$

where $\delta_{i, j}=1$ when $i=j$ and zero otherwise.

(iii) $\left[a d_{-f}^{k} r_{i}, a d_{-f}^{l} r_{j}\right]=0,1 \leq i, j \leq 2 ; 0 \leq k \leq k_{i}-$ $1 ; 0 \leq l \leq k_{j}-1$.

If the conditions of Theorem 1 hold for all $\zeta \in \mathbb{R}^{n}$ and the $n$ vector fields in Condition (iii) are complete, the OF coordinates are globally defined. One approach to deriving the $\mathrm{OF}$ state coordinates which include an output transformation is to use Theorem 1 with a general output function $\Psi(y)$ instead of the original function $h$. Without an output transformation, Condition (i) is not satisfied for $b_{2}-b_{3} \xi_{2} \neq 0$. This can be seen from $R_{1}^{l}, R_{1}^{r}$ :

$$
\begin{aligned}
& R_{1}^{l}=\left[\begin{array}{c}
d h_{1} \\
d h_{2} \\
d L_{f} h_{2}
\end{array}\right]=\left[\begin{array}{ccc}
1 & 0 & 0 \\
0 & 1 & 0 \\
* & * & b_{2}-b_{3} \xi_{2}
\end{array}\right] \\
& R_{1}^{r}=\left[\begin{array}{l}
d h_{1} \\
d h_{2}
\end{array}\right]=\left[\begin{array}{lll}
1 & 0 & 0 \\
0 & 1 & 0
\end{array}\right],
\end{aligned}
$$


which have different dimension unless $b_{2}-b_{3} \xi_{2}=0$. The dimensions of $R_{1}^{l}, R_{1}^{r}$ can be made equal if we transform the second output: $\bar{y}=\left[\bar{y}_{1}, \bar{y}_{2}\right]^{T}=\left[\xi_{1}, \Psi_{2}\left(\xi_{1}, \xi_{2}\right)\right]^{T}$. Relative to this output we have

$$
R_{1}^{l}=\left[\begin{array}{ccc}
1 & 0 & 0 \\
\frac{\partial \Psi_{2}}{\partial \xi_{1}} & \frac{\partial \Psi_{2}}{\partial \xi_{2}} & 0 \\
* & * & \frac{\partial \Psi_{2}}{\partial \xi_{1}}\left(b_{1}-b_{3} \xi_{1}\right)+\frac{\partial \Psi_{2}}{\partial \xi_{2}}\left(b_{2}-b_{3} \xi_{2}\right)
\end{array}\right]
$$

The condition $\operatorname{dim} R_{1}^{l}=\operatorname{dim} R_{1}^{r}$ yields a PDE

$$
\frac{\partial \Psi_{2}}{\partial \xi_{1}}\left(b_{1}-b_{3} \xi_{1}\right)+\frac{\partial \Psi_{2}}{\partial \xi_{2}}\left(b_{2}-b_{3} \xi_{2}\right)=0
$$

whose general solution is

$$
\Psi_{2}\left(\xi_{1}, \xi_{2}\right)=F\left(\frac{b_{3} \xi_{2}-b_{2}}{b_{3}\left(b_{3} \xi_{1}-b_{1}\right)}\right)
$$

We choose $F$ as the identity function:

$$
\Psi_{2}\left(\xi_{1}, \xi_{2}\right)=\frac{b_{3} \xi_{2}-b_{2}}{b_{3}\left(b_{3} \xi_{1}-b_{1}\right)}
$$

We remark that this choice restricts $b_{3} \neq 0$ for $\Psi_{2}$ to be well-defined. Other choices for $\Psi_{2}$ were investigated in order to eliminate this condition on the motion parameters. In all cases, varying $\Psi_{2}$ led to points of singularity at certain values of the motion parameters.

Next, we introduce the first component for the output transformation which for simplicity is taken to be a function of $\xi_{1}$ alone: $\bar{y}=\left[\bar{y}_{1}, \bar{y}_{2}\right]^{T}=\left[\Psi_{1}\left(\xi_{1}\right), \Psi_{2}\left(\xi_{1}, \xi_{2}\right)\right]^{T}$. Using Condition (ii) we obtain a non-unique solution for $r_{2}$ and an $r_{1}$ dependent on $\frac{d \Psi_{1}}{d y_{1}}$ :

$r_{1}=\frac{1}{\left(b_{1}-b_{3} \xi_{1}\right) \frac{d \Psi_{1}}{d \xi_{1}}} \frac{\partial}{\partial \xi_{3}}, r_{2}=\left(b_{3} \xi_{1}-b_{1}\right) \frac{\partial}{\partial \xi_{2}}+\rho\left(\xi_{1}\right) \frac{\partial}{\partial \xi_{3}}$,

where we have assumed $\rho\left(\xi_{1}\right)$ is some function of $\xi_{1}$ to be determined. We remark that for systems with all observability indices equal, then no such degree of freedom results from Condition (ii).

Next, we compute $a d_{-f} r_{1}$ and verify the Lie bracket Condition (iii). The condition $\left[r_{1}, r_{2}\right]=0$ holds for all $\xi \in \mathbb{R}^{3}$ except $b_{3} \xi_{1}-b_{1}=0$. The condition $\left[r_{1}, a d_{-f} r_{1}\right]=$ 0 if

$$
\frac{d^{2} \Psi_{1}}{d \xi_{1}^{2}}\left(b_{1}-b_{3} \xi_{1}\right)-2 b_{3} \frac{d \Psi_{1}}{d \xi_{1}}=0 .
$$

Solving this ODE gives

$$
\Psi_{1}\left(\xi_{1}\right)=C_{1}+\frac{C_{2}}{b_{3} \xi_{1}-b_{1}}
$$

where we choose $C_{1}=0$ and $C_{2}=1$. Hence,

$$
\bar{y}_{1}=\frac{1}{b_{3} \xi_{1}-b_{1}}, \quad \bar{y}_{2}=\frac{b_{3} \xi_{2}-b_{2}}{b_{3}\left(b_{3} \xi_{1}-b_{1}\right)} .
$$

Using this $\Psi_{1}$ the condition $\left[r_{2}, a d_{-f} r_{1}\right]=0$ requires

$$
\frac{d \rho}{d \xi_{1}}\left(b_{3} \xi_{1}-b_{1}\right)+a_{32} b_{1}-b_{3} a_{12}-\rho\left(\xi_{1}\right) b_{3}=0
$$

Solving this ODE gives

$$
\rho\left(\xi_{1}\right)=\left(b_{3} \xi_{1}-b_{1}\right) C_{3}+\left(a_{32} b_{1}-b_{3} a_{12}\right) / b_{3},
$$

and choosing $C_{3}=0$ gives $\rho=\left(a_{32} b_{1}-b_{3} a_{12}\right) / b_{3}$. The state transformation is $z=\Phi(\xi)=\left[\Phi_{1}(\xi), \Psi_{1}(\xi), \Psi_{2}(\xi)\right]^{T}$ with $\Phi_{1}$ given by

$$
\begin{aligned}
& \Phi_{1}(\xi)=\frac{c_{1}\left(\xi_{1}\right) \xi_{3}+c_{2} \xi_{2}+c_{3} \xi_{1}+c_{4}}{2 b_{3}\left(b_{3} \xi_{1}-b_{1}\right)^{2}} \\
& c_{1}\left(\xi_{1}\right)=2 b_{3}^{2}\left(b_{3} \xi_{1}-b_{1}\right) \\
& c_{2}=2 b_{3}\left(a_{12} b_{3}-a_{32} b_{1}\right) \\
& c_{3}=2 b_{3}\left(a_{11} b_{3}-a_{31} b_{1}\right) \\
& c_{4}=b_{3}\left(b_{3} a_{13}-\left(a_{33}+a_{11}\right) b_{1}-b_{2} a_{12}\right)+a_{31} b_{1}^{2}+b_{2} a_{32} b_{1} .
\end{aligned}
$$

Applying this state transformation and the output transformation (5) gives the $\mathrm{OF}$

$$
\dot{z}=\left[\begin{array}{c}
\eta_{1}(\bar{y}) \\
z_{1}+\eta_{2}(\bar{y}) \\
\eta_{3}(\bar{y})
\end{array}\right], \quad \bar{y}=\left[\begin{array}{c}
z_{2} \\
z_{3}
\end{array}\right]
$$

The functions $\eta_{i}, i=1,2,3$ are polynomials:

$$
\begin{aligned}
\eta_{1}(\bar{y}) & =d_{11} \bar{y}_{2}^{3}+d_{12} \bar{y}_{2} \bar{y}_{1}^{2}+d_{13} \bar{y}_{1} \bar{y}_{2}^{2}+d_{14} \bar{y}_{1}^{2}+d_{15} \bar{y}_{2}^{2} \\
& +d_{16} \bar{y}_{1} \bar{y}_{2}+d_{17} \bar{y}_{1}+d_{18} \bar{y}_{2}+d_{19} \\
\eta_{2}(\bar{y}) & =d_{21} \bar{y}_{1}^{2}+d_{22} \bar{y}_{1} \bar{y}_{2}+d_{23} \bar{y}_{1}+d_{24} \bar{y}_{2}+d_{25} \\
\eta_{3}(\bar{y}) & =d_{31} \bar{y}_{2}^{2}+d_{32} \bar{y}_{1} \bar{y}_{2}+d_{33} \bar{y}_{1}+d_{34} \bar{y}_{2}+d_{35},
\end{aligned}
$$

where $d_{i j}$ are constants depending on $b_{i}, a_{i j}, 1 \leq i, j \leq 3$.

An observer for (2) can now be constructed as

$$
\dot{\hat{\zeta}}=f(\hat{\zeta})+\frac{\partial \zeta}{\partial z}(\hat{\zeta})(\eta(\bar{y})-\eta(\Psi(h(\hat{\zeta})))+K(\bar{y}-\Psi(h(\hat{\zeta})))
$$

and we introduce parameters $\omega_{p}, \zeta_{p}$, and $\alpha_{p}$ and $K=$ $\left[\omega_{p}^{2}, 2 \zeta_{p} \omega_{p}, \alpha_{p}\right]^{T}$ so that the characteristic equation for the error dynamics of $(7)$ in the $z$-coordinates is

$$
\left(s+\alpha_{p}\right)\left(s^{2}+2 \zeta_{p} \omega_{p} s+\omega_{p}^{2}\right)=0 .
$$


This allows us to directly control the rate of convergence of the estimate error and represents an important design feature of the proposed method.

The state transformation $z=\Phi(\xi)$ and the output transformation $\bar{y}=\Psi(y)$ require $b_{3} \neq 0$. However, the case $b_{3}=0$ can be handled using the same procedure using a linear output transformation $\bar{y}_{1}=\xi_{1}, \bar{y}_{2}=b_{1} \xi_{2}-b_{2} \xi_{1}$, which is valid when $b_{1} \neq 0$. For the case $b_{1}=b_{3}=0$, no output transformation is required [5].

The above approach can also be applied to the planar perspective system

$$
\dot{\zeta}=\left[\begin{array}{ll}
a_{11} & a_{12} \\
a_{21} & a_{22}
\end{array}\right] \zeta+\left[\begin{array}{l}
b_{1} \\
b_{2}
\end{array}\right], \quad y=\frac{\zeta_{1}}{\zeta_{2}},
$$

which does not admit an OF without output transformation [18]. The details of the procedure are straightforward and not provided. It is also interesting to note that since the system is two dimensional, it is transformable to $\mathrm{OF}$ using a time scale transformation.

The method described above to compute the OF coordinates uses Theorem 1. Alternatively, one can use a method based on a so-called Generalized Characteristic Equation (GCE) [12]. For a two output system with observability indices $(2,1)$, the GCEs are

$$
L_{f}^{2} \Psi_{1}(y)=L_{f} \eta_{2}(\Psi(y))+\eta_{1}(\Psi(y)), L_{f} \Psi_{2}(y)=\eta_{3}(\Psi(y))
$$

Expanding the GCEs and performing coefficient matching leads to necessary and sufficient conditions on the transformability to OF. In particular the so-called polynomial condition results: $\partial^{2} L_{f}^{2} \Psi_{1}(y) / \partial \dot{y}_{1}^{2}=0$ and $\partial L_{f} \Psi_{2}(y) / \partial \dot{y}_{1}=0$. We assume the output transformation for the first subsystem to only depend on $y_{1}$, i.e. $\bar{y}_{1}=\Psi_{1}\left(y_{1}\right)$. We are able to solve for $\Psi_{1}$ s.t. the system satisfies a polynomial condition. That is, $L_{f}^{2} \bar{y}_{1}$ is linear in $\dot{y}_{1}$ with coefficients depending on $y$ :

$$
\ddot{\bar{y}}_{1}=\frac{d^{2} \Psi_{1}}{d y_{1}^{2}} \dot{y}_{1}^{2}+\frac{d \Psi_{1}}{d y_{1}}\left(\alpha_{2}(y) \dot{y}_{1}^{2}+\alpha_{3}(y) \dot{y}_{1}+\alpha_{4}(y)\right)
$$

In order to remove the dependence on $\dot{y}_{1}^{2}$ on the RHS of (9) we have the ODE

$$
\frac{d^{2} \Psi_{1}}{d y_{1}^{2}}+\alpha_{2}(y) \frac{d \Psi_{1}}{d y_{1}}=0, \quad \alpha_{2}(y)=\frac{2 b_{3}}{b_{3} y_{1}-b_{1}}
$$

We notice that ODE (10) is the same as (4), hence the GCE approach leads to the same $\Psi_{1}$ as computed above. For the second subsystem we assume a more general dependence for the output transformation: $\Psi_{2}(y)$. Following the similar procedure as that used for the first sub- system we have

$\dot{\bar{y}}_{2}=\frac{\partial \Psi_{2}}{\partial y_{1}} \dot{y}_{1}+\frac{\partial \Psi_{2}}{\partial y_{2}} \dot{y}_{2}=\frac{\partial \Psi_{2}}{\partial y_{1}} \dot{y}_{1}+\frac{\partial \Psi_{2}}{\partial y_{2}}\left(\alpha_{5}(y) \dot{y}_{1}+\alpha_{6}\left(y_{1}\right)\right)$

and the PDE

$$
\frac{\partial \Psi_{2}}{\partial y_{1}}+\frac{\partial \Psi_{2}}{\partial y_{2}} \alpha_{5}(y)=0, \quad \alpha_{5}(y)=\frac{b_{3} y_{2}-b_{2}}{b_{3} y_{1}-b_{1}} .
$$

One can see that PDE (11) is the same as (3). Hence, the GCE approach yields the same output transformation $\Psi_{2}(y)$ as computed above.

\subsection{Time-scaled block triangular observer form}

The system (2) in observable form is already in BTF [20]. We attempt to transform the first subsystem to BTOF [19]. Defining the observable coordinates as $x=$ $\left[x_{1}^{T}, x_{21}\right]^{T}=\left[x_{11}, x_{12}, x_{21}\right]^{T}=\left[h_{1}(\xi), L_{f} h_{1}(\xi), h_{2}(\xi)\right]^{T}$, one can compute the starting vector $g_{1}=\partial / \partial x_{12}$ according to [21, Eq. (6)] and verify that the Lie bracket $[21$, Eq. (7)] is not satisfied. We introduce the time scaling transformation for the first subsystem

$$
\frac{d \tau_{1}}{d t}=s_{1}(y)>0
$$

where $s_{1}(y)$ is the time scaling function (TSF) to be determined. We apply [21, Prop. 3.1]

$$
d L_{g_{1}} L_{F^{1}}^{k_{1}} h_{1}=l_{k_{1}} \frac{1}{s_{1}} \frac{\partial s_{1}}{\partial y_{1}} d L_{F^{1}} h_{1} \quad \bmod \left\{d z_{1}^{1}\right\}
$$

with $k_{1}=2, F^{1}=f_{1}=x_{12} \partial / \partial x_{11}+\left(L_{f}^{2} h_{1}(x)\right) \partial / \partial x_{12}$, and $l_{2}=2$. This yields the PDE for $s_{1}$

$$
\frac{4 b_{3}}{b_{3} y_{1}-b_{1}}=\frac{2}{s_{1}} \frac{\partial s_{1}}{\partial y_{1}} .
$$

Solving this PDE yields the time scaling transformation

$$
\frac{d \tau_{1}}{d t}=\left(b_{3} y_{1}-b_{1}\right)^{2}=s_{1}(y)>0
$$

Defining $\bar{f}_{1}=f_{1} / s_{1}$ and calculating the vector fields $\bar{g}_{1}=s_{1} g_{1}, a d_{-\bar{f}_{1}} \bar{g}_{1}$, we can verify the Lie bracket condition $\left[\bar{g}_{1}, a d_{-\bar{f}_{1}} \bar{g}_{1}\right]=0$. However, [21, Eq. (8)] requires $\frac{\partial}{\partial y_{2}} a d_{-f_{1}} \bar{g}_{1}=0$ which is satisfied if and only if

$$
a_{12} b_{3}-a_{32} b_{1}=0
$$

This constraint also appears in the dynamic error linearization in [5]. Given (12), the transformation of state 
can be solved from $\frac{\partial \Phi_{1}\left(x_{1}\right)}{\partial x_{1}}\left[a d_{-\bar{f}_{1}} \bar{g}_{1}, \bar{g}_{1}\right]=I_{2}$, where $I_{2}$ is an identity matrix and

$$
\begin{aligned}
& a d_{-\bar{f}_{1}} \bar{g}_{1}=\left[\begin{array}{c}
1 \\
\frac{-2 b_{3} x_{12}+c_{2} x_{11}+c_{3}}{b_{1}-b_{3} x_{11}}
\end{array}\right] \\
& c_{3}=-2 a_{33} b_{1}+3 b_{3} a_{13}+a_{11} b_{1}+a_{12} b_{2} \\
& c_{2}=2 b_{3} a_{11}-3 a_{31} b_{1}-a_{32} b_{2}-a_{33} b_{3} .
\end{aligned}
$$

This gives the transformation to TBTOF:

$$
z=\left[\begin{array}{c}
z_{11} \\
z_{12} \\
z_{21}
\end{array}\right]=\left[\begin{array}{c}
\Phi_{1}(x) \\
\Phi_{2}(x) \\
\Phi_{3}(x)
\end{array}\right]=\left[\begin{array}{c}
x_{11} \\
\frac{2 x_{12} b_{3}^{2}+c_{2} b_{1}-c_{3} b_{3}-2 c_{2} x_{11} b_{3}}{2 b_{3}^{2}\left(b_{1}-b_{3} x_{11}\right)^{2}} \\
x_{21}
\end{array}\right]
$$

where we have reused the notation for $z$ and $\Phi$. In $z$ coordinates the system is

$$
\left[\begin{array}{c}
\frac{d z_{11}}{d \tau_{1}} \\
\frac{d z_{12}}{d \tau_{1}} \\
\frac{d z_{21}}{d t}
\end{array}\right]=\left[\begin{array}{c}
z_{12}+\beta_{11}\left(z_{11}, y_{2}\right) \\
\beta_{12}\left(z_{11}, y_{2}\right) \\
\beta_{21}\left(z_{1}, z_{21}\right)
\end{array}\right], \quad y=\left[\begin{array}{c}
z_{11} \\
z_{21}
\end{array}\right]
$$

The TBTOF allows for a straightforward observer design

$$
\left[\begin{array}{c}
\frac{d \hat{z}_{1}}{d \tau_{1}} \\
\frac{d \hat{z}_{21}}{d t}
\end{array}\right]=\left[\begin{array}{c}
A_{1} \hat{z}_{1}+\beta_{1}+L_{1} C_{1}\left(z_{1}-\hat{z}_{1}\right) \\
\hat{\beta}_{21}+L_{2}\left(z_{21}-\hat{z}_{21}\right)
\end{array}\right]
$$

where $\hat{z}_{1}=\left[\hat{z}_{11}, \hat{z}_{12}\right]^{T}, A_{1}=\left[\begin{array}{ll}0 & 1 \\ 0 & 0\end{array}\right], C_{1}=[1,0]^{T}, \beta_{1}=$ $\left[\beta_{11}, \beta_{12}\right]^{T}, \hat{\beta}_{21}=\beta_{21}\left(\hat{z}_{12}, y\right)$, and $L_{1}$ is chosen so that $A_{1}-L_{1} C_{1}$ is Hurwitz, and $L_{2}>0$. The corresponding error dynamics in the new time scale is

$$
\left[\begin{array}{c}
\frac{d \tilde{z}_{1}}{d \tau_{1}} \\
\frac{d \tilde{z}_{21}}{d t}
\end{array}\right]=\left[\begin{array}{cc}
A_{1}-L_{1} C_{1} & 0 \\
0 & -L_{2}
\end{array}\right] \tilde{z}+\left[\begin{array}{c}
0 \\
\beta_{21}-\hat{\beta}_{21}
\end{array}\right]
$$

whose zero solution is globally exponentially stable (GES) assuming $b_{3} x_{11}-b_{1} \neq 0$ and $\beta_{21}$ globally Lipschitz in $z_{12}$. Assuming there exist positive constants $T_{0}, \varepsilon$ such that

$$
\int_{t}^{t+T_{0}} s_{1}(\xi) \mathrm{d} \xi \geq \varepsilon, \quad \forall t \geq t_{0}
$$

we conclude the zero solution of the error dynamics is GES in the original time. The observer in $x$-coordinates and $t$ time is

$$
\begin{aligned}
\dot{\hat{x}}= & {\left[\begin{array}{c}
\frac{s_{1}(y)}{s_{1}(\hat{y})} f_{1}(\hat{x}) \\
f_{2}(\hat{x})
\end{array}\right] } \\
& +\left(\frac{\partial \hat{z}}{\partial \hat{x}}\right)^{-1}\left[\begin{array}{c}
s_{1}(y)\left(\beta_{1}-\hat{\beta}_{1}^{*}+L_{1}\left(y_{1}-C_{1} \hat{z}_{1}\right)\right) \\
\hat{\beta}_{21}-\hat{\beta}_{21}^{*}+L_{2}\left(y_{2}-\hat{z}_{21}\right)
\end{array}\right],
\end{aligned}
$$

where $f_{2}$ is the third component of the dynamics in observable form, $\hat{\beta}_{1}^{*}=\beta_{1}\left(\hat{z}_{11}, \hat{y}_{2}\right), \hat{\beta}_{21}^{*}=\beta_{21}\left(\hat{z}_{1}, \hat{y}_{2}\right)$. The TBTOF coordinates involve the transformation of a 2dimensional subsystem of the perspective dynamics. It does not transform the 2nd subsystem dynamics, i.e., the dynamics of $y_{2}=x_{21}=z_{21}=\zeta_{2} / \zeta_{3}$. Hence, the advantage this design has over the OF-based design is a simpler means of construction and a simpler expression for the change of coordinates and observer. This simplicity has the practical benefit of reduced sensitivity to motion parameter error.

\section{Simulations}

We simulate the OF and TBTOF observers for the system

$$
\dot{\zeta}=\left[\begin{array}{ccc}
0 & -1 & 1 \\
1 & 0 & 1 \\
-1 & -1 & 0
\end{array}\right] \zeta+\left[\begin{array}{l}
1 \\
2 \\
1
\end{array}\right]
$$

The eigenvalues of error dynamics are placed at $-4(\mathrm{OF})$ and -2.5 (TBTOF), and the initial conditions (ICs) in the format of $\left[\zeta_{1}, \zeta_{2}, \zeta_{3}, \hat{\zeta}_{1}, \hat{\zeta}_{2}, \hat{\zeta}_{3}\right]^{T}$ are

$$
\begin{aligned}
& I C 1:[-1,2,2,-1 / 6,1 / 3,1 / 3]^{T} \\
& I C 2:[-1,2,1,-0.03,0.12,0.30]^{T} \\
& I C 3:[-2,3,4,-0.4,2.4,0.4]^{T} .
\end{aligned}
$$

For the OF-based observer, a plot of the norm of the error in the original coordinates $\|\tilde{\zeta}\|=\|\zeta-\hat{\zeta}\|$ is presented in Figure 1, using solid (IC1), dashed (IC2), and dashed-dot (IC3) lines for the initial conditions in (13). For the TBTOF-based observer, the corresponding simulation result is given in Figure 2. Uniformly distributed noise with an amplitude of .005 was added to the output to demonstrate the designs' robustness to measurement noise. The TBTOF-based design exhibits more noise in the estimate error which can be due to the faster error convergence.

To compare the OF-based design with an existing ap- 


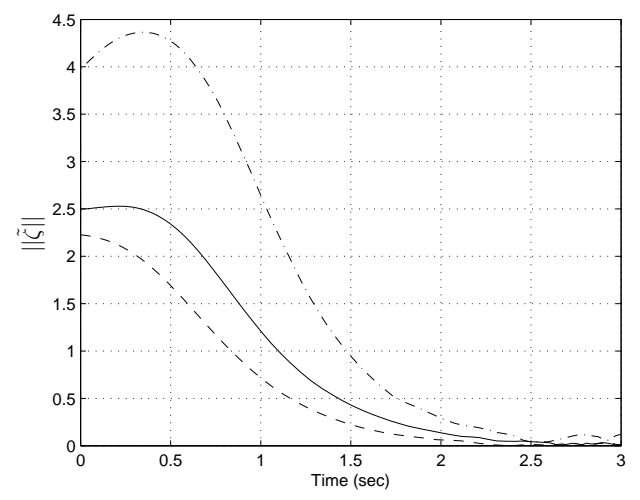

Fig. 1. Norm of state estimate error in $\zeta$-coordinates using the OF-based design.

proach in [11], we consider the example in $[2,9,11]$ :

$$
\dot{\zeta}=\left[\begin{array}{ccc}
-0.2 & 0.4 & -0.6 \\
0.1 & -0.2 & 0.3 \\
0.3 & -0.4 & 0.4
\end{array}\right] \zeta+\left[\begin{array}{c}
0.5 \\
0.25 \\
0.3
\end{array}\right],
$$

with the initial conditions as in $[11]:\left[\zeta_{1}(0), \zeta_{2}(0), \zeta_{3}(0)\right]^{T}=$ $[1,1.5,2.5]^{T},\left[\hat{\zeta}_{1}(0), \hat{\zeta}_{2}(0), \hat{\zeta}_{3}(0)\right]^{T}=[0,0,1]^{T}$. Simulations for three different sets of eigenvalues demonstrate the control of the rate of convergence of the estimate error: we take the parameters $\alpha_{p}=\omega_{p}=4,8,12$ and $\zeta_{p}=1$ in (8) which roughly compare with convergence corresponding to $\lambda=10,20,30$ in [11]. The simulation results for $\tilde{\zeta}_{3}$ for both observers for no measurement noise are in Figure 3. To investigate the proposed design's ability to trade off robustness to noise with speed of convergence, uniformly distributed noise with an amplitude of .005 was added to the output of both designs. The simulation results for $\tilde{\zeta}_{3}$ are shown in Figure 4. We remark the OF-based design has favorable noise rejection properties relative to the design in [11]. This is because the term $\beta(y)$ in the expression for $\hat{\zeta}_{3}$ which can directly magnify the noise. Similarly, a large value of $\lambda$ can amplify noise. The design in [11] is also reducedorder which implies estimates for $\zeta_{1}$ and $\zeta_{2}$ will depend directly on measurement noise. Further, the parameter $\lambda$ in [11] requires knowledge of a bound on the size of system's state which makes the design of the rate of error convergence less direct relative to the OF-based design.

\section{Conclusions}

This paper has shown that a perspective system admits two observer forms. These observer forms naturally lead to observer designs with error dynamics which are easy to stabilize. The first observer form is the OF with output transformation which provides error convergence without motion constraints (assuming constant motion

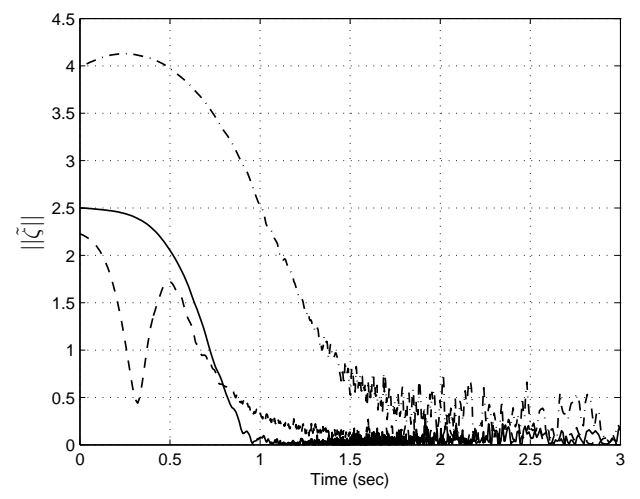

Fig. 2. Norm of state estimate error in $\zeta$-coordinates using the TBTOF-based design.
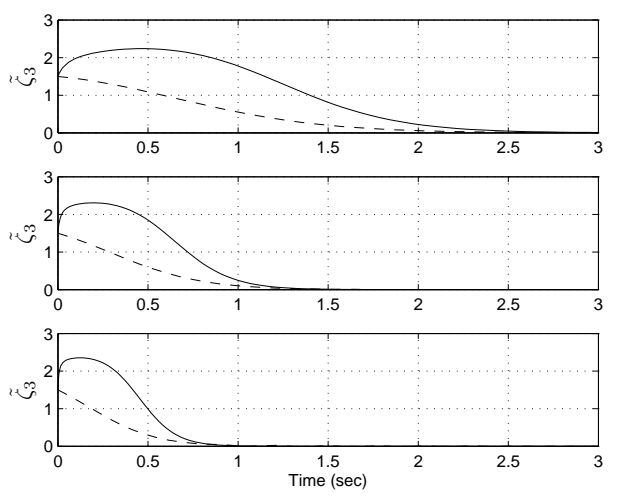

Fig. 3. Solid curves are state estimate error $\tilde{\zeta}_{3}$ using an OF design for three choices of eigenvalues: -4 (top), -8 (middle), and -12 (bottom). Dashed curves are observer in [11] for $\lambda=10($ top $), 20($ middle $), 30($ bottom $)$.
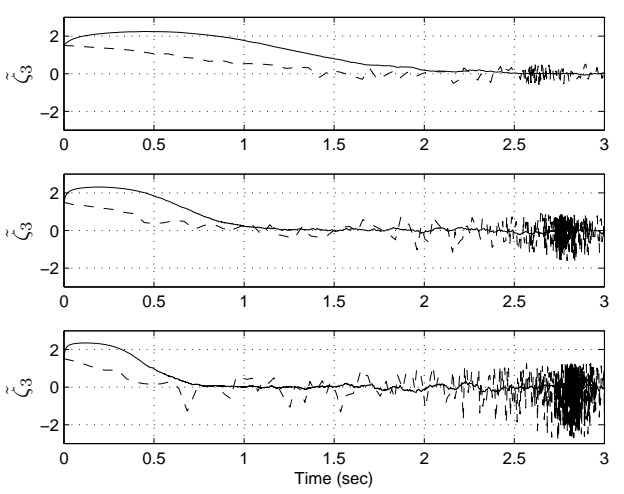

Fig. 4. Solid curves are state estimate error $\tilde{\zeta}_{3}$ using an OF design for three choices of eigenvalues: -4 (top), -8 (middle), and -12 (bottom). Dashed curves are observer in [11] for $\lambda=10$ (top), 20(middle), 30(bottom). Noise was added to the output measurements. 
parameters). The second observer form is a TBTOF which requires the same motion constraint as in previous work [5] on dynamic error linearization but has the advantage of a relatively simple observer structure. Future work involves generalizing the normal form-based approach to allow for time-varying and/or unknown motion parameters.

\section{References}

[1] R. Abdursul, H. Inaba, and B. K. Ghosh. Nonlinear observers for perspective time-invariant linear systems. Automatica, 40:481-490, 2004.

[2] X. Chen and H. Kano. A new state observer for perspective systems. IEEE Transactions on Automatic Control, 47(4):658-663, 2002.

[3] X. Chen and H. Kano. State observer for a class of nonlinear systems and its application to machine vision. IEEE Transactions on Automatic Control, 49(11):2085-2091, 2004.

[4] O. Dahl. A Maple function library for observer error linearization, 2008. Technical Report, Malmö University.

[5] O. Dahl, F. Nyberg, and A. Heyden. On observer error linearization for perspective dynamic systems. In American Control Conference, July 2007.

[6] O. Dahl, F. Nyberg, and A. Heyden. Structure and motion estimation in perspective systems using a dynamic vision parametrization. In European Control Conference, July 2007.

[7] O. Dahl, F. Nyberg, J. Holst, and A. Heyden. Linear design of a nonlinear observer for perspective systems. In Proc. of ICRA'05 - 2005 IEEE Conference on Robotics and Automation, April 2005.

[8] O. Dahl, Y. Wang, A. F. Lynch, and A. Heyden. Observer forms for perspective systems. In 17th IFAC World Congress, pages 8618-8623, Seoul, Korea, Jul. 2008.

[9] W. E. Dixon, Y. Fang, D. M. Dawson, and T. J. Flynn. Range identification for perspective vision systems. IEEE Transactions on Automatic Control, 48(12):2232-2238, 2003.

[10] M. Jankovic and B. K. Ghosh. Visually guided ranging from observations of points, lines and curves via an identifier based nonlinear observer. Systems $\&$ Control Letters, 25:63-73, 1995.

[11] D. Karagiannis and A. Astolfi. A new solution to the problem of range identification in perspective vision systems. IEEE Transactions on Automatic Control, 50(12):2074-2077, 2005.

[12] H. Keller. Nonlinear observer design by transformation into a generalized observer canonical form. International Journal of Control, 46(6):1915-1930, 1987.

[13] A. J. Krener and W. Respondek. Nonlinear observers with linearizable error dynamics. SIAM Journal on Control and Optimization, 23(2):197216, 1985.

[14] Y. Ma, S. Soatto, J. Košecká, and S. S. Sastry. An Invitation to 3-D Vision. Springer-Verlag, 2004.

[15] L. Matthies, T. Kanade, and R. Szeliski. Kalman filter-based algorithms for estimating depth from image sequences. International Journal of Computer Vision, 3:209-236, 1989.

[16] A. Matveev, X. Hu, R. Frezza, and H. Rehbinder. Observers for systems with implicit output. IEEE Transactions on Automatic Control, 45(1):168-173, 2000.

[17] S. Soatto, R. Frezza, and P. Perona. Motion estimation via dynamic vision. IEEE Transactions on Automatic Control, 41(3):393-413, 1996.

[18] S. Soatto and P. Perona. On the exact linearization of structure from motion. Technical Report CIT-CDS 94-011, California Institute of Technology, June 1994.

[19] Y. Wang and A. Lynch. A block triangular observer forms for nonlinear observer design. International Journal of Control, 81(2):177-188, 2008.

[20] Y. Wang and A. F. Lynch. A block triangular form for nonlinear observer design. IEEE Transactions on Automatic Control, 51(11):1803-1808, 2006.

[21] Y. Wang and A. F. Lynch. Observer design using a time scaled block triangular observer form. In American Control Conference, pages 799-804, Minneapolis, MN, June 2006.

[22] X.-H. Xia and W.-B. Gao. Nonlinear observer design by observer error linearization. SIAM Journal on Control and Optimization, 27(1):199-216, 1989. 